\title{
High-Quality Ultrathin Gold Layers For Use In Plasmonic And Metamaterials
} Applications

Sukham, Johneph; Takayama, Osamu; Lavrinenko, Andrei; Malureanu, Radu

Published in:

Proceedings of SPIE

Link to article, DOI:

$10.1117 / 12.2306135$

Publication date:

2018

Document Version

Publisher's PDF, also known as Version of record

Link back to DTU Orbit

Citation (APA):

Sukham, J., Takayama, O., Lavrinenko, A., \& Malureanu, R. (2018). High-Quality Ultrathin Gold Layers For Use In Plasmonic And Metamaterials Applications. In Proceedings of SPIE (Vol. 10671). [1067111] SPIE -

International Society for Optical Engineering. Proceedings of SPIE - The International Society for Optical Engineering https://doi.org/10.1117/12.2306135

\section{General rights}

Copyright and moral rights for the publications made accessible in the public portal are retained by the authors and/or other copyright owners and it is a condition of accessing publications that users recognise and abide by the legal requirements associated with these rights.

- Users may download and print one copy of any publication from the public portal for the purpose of private study or research.

- You may not further distribute the material or use it for any profit-making activity or commercial gain

- You may freely distribute the URL identifying the publication in the public portal 


\section{High-quality ultrathin gold layers for use in plasmonic and metamaterials applications}

Johneph Sukham, Osamu Takayama, Andrei V. Lavrinenko, Radu Malureanu

Johneph Sukham, Osamu Takayama, Andrei V. Lavrinenko, Radu Malureanu, "High-quality ultrathin gold layers for use in plasmonic and metamaterials applications ," Proc. SPIE 10671, Metamaterials XI, 1067111 (7 May 2018); doi: 10.1117/12.2306135

SPIE. Event: SPIE Photonics Europe, 2018, Strasbourg, France 


\title{
High-Quality Ultrathin Gold Layers For Use In Plasmonic And Metamaterials Applications
}

\author{
Johneph Sukham ${ }^{\mathrm{a}}$, Osamu Takayama ${ }^{\mathrm{a}}$, Andrei V. Lavrinenko ${ }^{\mathrm{a}}$, and Radu Malureanu ${ }^{\mathrm{a}, \mathrm{b}}$ \\ ${ }^{a}$ Technical University of Denmark, Dept. of Photonics Engineering, Oersteds plads, bldg. \\ 345A, DK-2800, Kgs. Lyngby, Denmark \\ ${ }^{b}$ Technical University of Denmark, National Centre for Micro- and Nanofabrication, Oersteds \\ plads, bldg. 347, DK-2800, Kgs. Lyngby, Denmark
}

\begin{abstract}
The propagation of electromagnetic waves can be manipulated at the nanoscale by surface plasmons supported by ultra thin metal layers. An adhesion layer, with thickness in the order of few nanometerss is used for depositing ultra thin metal gold layers. Cr and $\mathrm{Ti}$ are the most popular metallic adhesion layers. Apart from them, a non metallic silane based wetting layer like (3-Aminopropyl)trimethoxysilane (APTMS) can be used. The behaviour of the propagating surface plasmons due to the influence of these adhesion layers has not been thoroughly investigated. To study the influence of the adhesion layers on propagating plasmons for use in plasmonic and metamaterial applications, we experimentally compared the performances of the ultra-thin gold layers using $\mathrm{Cr}$ and APTMS adhesion layers and without any adhesion layer. We show that the gold layers using APTMS adhesion exhibit short range surface plasmon polaritons (SR-SPPs) with characteristics close to the theoretical calculations, considering an ideal gold film.
\end{abstract}

Keywords: APTMS adhesion layer, ultra thin gold film, low roughness, plasmonics

\section{INTRODUCTION}

Plasmonics is playing a key role in the field of nanophotonics with a wide range of applications in nanofocussing, ${ }^{1}$ optical biosensing, ${ }^{2}$ enhanced emission of light. ${ }^{3}$ This is possible due to collective oscillations of electron plasma in the metal that have the ability to focus electromagnetic waves at nanoscale in metal-dielectric structures.

In the visible to near infra-red wavelengths regime, silver $(\mathrm{Ag})$ and gold $(\mathrm{Au})$ are known to be the best plasmonic materials due to their high plasma frequency. Au is mainly used in the field of plasmonics applications due to its chemical stability. The most commonly used adhesion layers for $\mathrm{Au}$ are $\mathrm{Ti}$ and $\mathrm{Cr}$ which are metallic but non metallic options like organosilane compounds ${ }^{4}$ can be used.The reported roughness of the Au layers using these different adhesion layers ranges from $0.30 \mathrm{~nm}$ to $1 \mathrm{~nm} .{ }^{5}$ However, even after successful fabrication of ultrathin films, introduction of these typically 1-2 $\mathrm{nm}$ thick metallic adhesion layers may affect the optical performance of the structures by introducing extra absorption or scattering of the localized surface plasmon polaritons.

One of the applications of ultra-thin Au film is the realization of anisotropic materials like hyperbolic metamaterials (HMMs). ${ }^{6}$ HMMs are used for various applications in nano imaging, biosensing and spontaneous emission engineering. ${ }^{7}$ Fabrication of multilayer HMMs in the visible and near infra red requires ultra-thin and ultra-smooth films with thicknesses in the range of $15 \mathrm{~nm}^{2}$ Therefore, the quest for adhesion layer to obtain thinner and smoother Au layers with non damping optical performance is crucial.

In this work, we show that silane based wetting layer, APTMS can achieve high quality ultra-thin gold layers with low roughness $0.30 \mathrm{~nm}$ rms which supports the short range surface plasmon polaritons with large wavevectors when compared experimentally with a $\mathrm{Cr}$ adhesion layer and without any adhesion layer.

Further author information: (Send correspondence to Radu Malureanu)

E-mail: rmal@dtu.dk

Metamaterials XI, edited by Allan D. Boardman, Anatoly V. Zayats, Kevin F. MacDonald, Proc. of SPIE

Vol. 10671, $1067111 \cdot$ @ 2018 SPIE · CCC code: 0277-786X/18/\$18 · doi: 10.1117/12.2306135 


\section{METHODS}

\subsection{Au Nanolayer Fabrication}

The samples are fabricated on $\mathrm{Si}$ wafers with thick $\mathrm{SiO}_{2}(8 \mu \mathrm{m})$. They are initially cleaned in Piranha solution $\left(70 \% \mathrm{H}_{2} \mathrm{SO}_{4}\right.$ and $30 \% \mathrm{H}_{2} \mathrm{O}_{2}$ ) for 20 minutes to remove any organic residues. The deposition of APTMS adhesion layers starts with the immersion into $2.5 \%$ APTMS in IPA solution for 3 hours. ${ }^{4,8}$ For the metallic ahdesion layer, $1 \mathrm{~nm}$ thick layer $\mathrm{Cr}$ are deposited using e-beam deposition at a very slow deposition rate of $2 \AA / \mathrm{s}$. Then, the $\mathrm{Au}$ layers with thicknesses of 8,10 and $18 \mathrm{~nm}$ are sputtered onto the samples with a constant deposition rate of $10 \AA / \mathrm{s}$. We deposited another $1 \mathrm{~nm}$ thick layer of APTMS and Cr respectively on top of the Au films

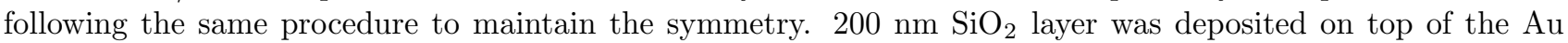
films using sputtering to maintain the symmetry of the mode and also to allow for excitation of plasmons using the Otto configuration.

The Au layer qualities are characterized using SEM and AFM. The various RMS roughness of the layers are listed in table 1. Fig 1 shows the SEM images for the Au layers with different adhesion layers with thickness of $8 \mathrm{~nm}$.

Table 1. RMS Roughness of Gold Layers with different thickness and adhesion layer

\begin{tabular}{|l|l|l|l|}
\hline & $\mathrm{Au}-8 \mathrm{~nm}$ & $\mathrm{Au}-10 \mathrm{~nm}$ & $\mathrm{Au}-18 \mathrm{~nm}$ \\
\hline 1nm APTMS & 0.30 & 0.31 & 0.32 \\
\hline 1nm Cr & 0.40 & 0.41 & 0.36 \\
\hline No adhesion & 1.24 & 0.63 & 0.51 \\
\hline
\end{tabular}

The Au films with the APTMS adhesion layer are the smoothest, with a root mean square (RMS) surface roughness of $0.30 \mathrm{~nm}$ followed by the Au films with the Cr adhesion layer with a surface roughness RMS of 0.40 $\mathrm{nm}$. From the SEM and AFM characterization, it is seen clearly that the Au without adhesion layer has the highest roughness of $1.2 \mathrm{~nm}$ rms and it can be correlated from the SEM image where the layer without adhesion layer has pin holes and cracks due to the percolation threshold limit of the gold layer.

\subsection{Optical Characterization}

The spectroscopic reflection measurements were performed using a ZnSe semi-cylinder as a high refractive index prism to excite SR-SPPs. The incidence angle was varied from 33 to 55 degrees with 2 degrees interval by rotating the mechanical stage. The light source was a NKT Photonics, super continuum broadband laser allowing for measurements between 600 and $1750 \mathrm{~nm}$ wavelength range. Each incidence angle was measured 10 times to eliminate the mechanical stage uncertainties: 5 times when increasing the incidence angle and 5 times when decreasing the angle. The reference of the reflection spectrum is the measurement of the reflected beam at 55 degrees incidence angle without sample to ensure total internal reflection (TIR).

\subsection{Numerical Simulation}

The simulations in Figure 2(a-c) are conducted by the scattering matrix formalism in order to calculate the reflectance from the multilayer structures. ${ }^{9}$ The model structure consists of 5 layers: $\mathrm{ZnSe}_{\text {prismSiO}}(200$ $\mathrm{nm}$ thick) $\mathrm{Au}$ nanofilm $(8,10,18 \mathrm{~nm}) \mathrm{SiO}_{2}(8 \mu \mathrm{m}) \mathrm{Si}$ substrate. The reflectance simulation was conducted with TM-polarized incident light with the wavelength range from $600 \mathrm{~nm}-1750 \mathrm{~nm}$ and the angle of incidence from $33^{\circ}-55^{\circ}$.

\section{RESULTS AND DISCUSSION}

Gold layers of thicknesses of 8,10 and $18 \mathrm{~nm}$ with three different configurations-with an APTMS adhesion layer, $\mathrm{Cr}$ adhesion layer and without any adhesion layer were fabricated to compare their performances.

We performed a series of reflection spectroscopic measurements to excite and detect SR-SPPs by using the Otto-configuration with high index ZnSe prism. The measured dispersion diagrams for SR-SPPs are obtained for 
a

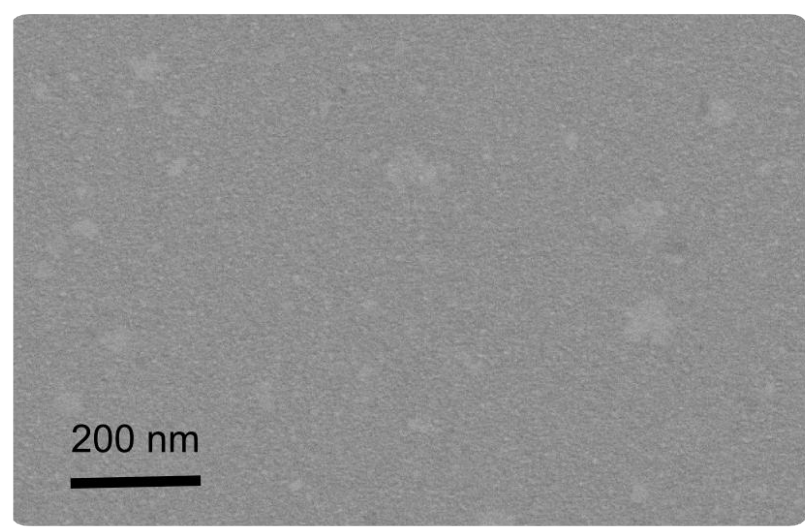

b

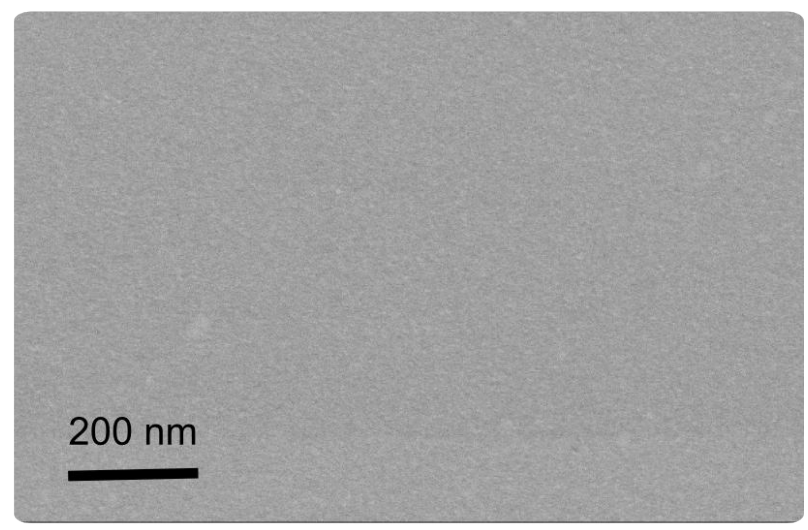

C

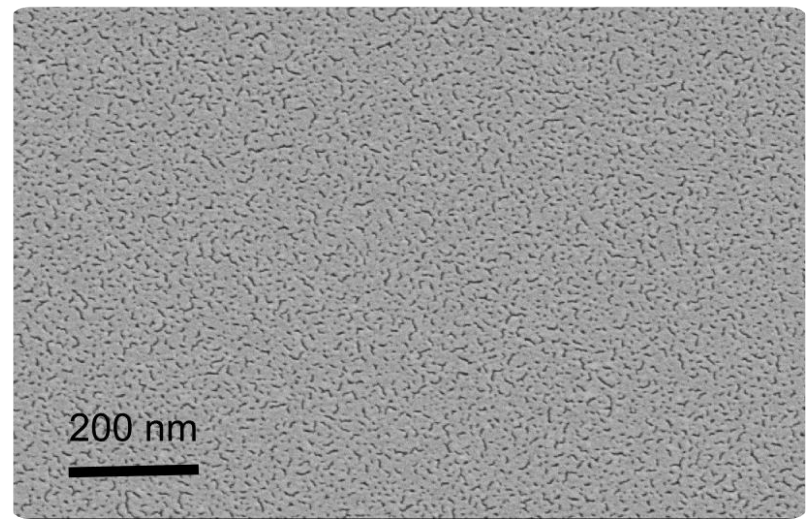

Figure 1. SEM images of (a)1 nm APTMS adhesion layer (b) $1 \mathrm{~nm} \mathrm{Cr}$ adhesion layer (c) and without adhesion layer for the thickness of $\mathrm{Au} 8 \mathrm{~nm}$

Proc. of SPIE Vol. 10671 1067111-3 

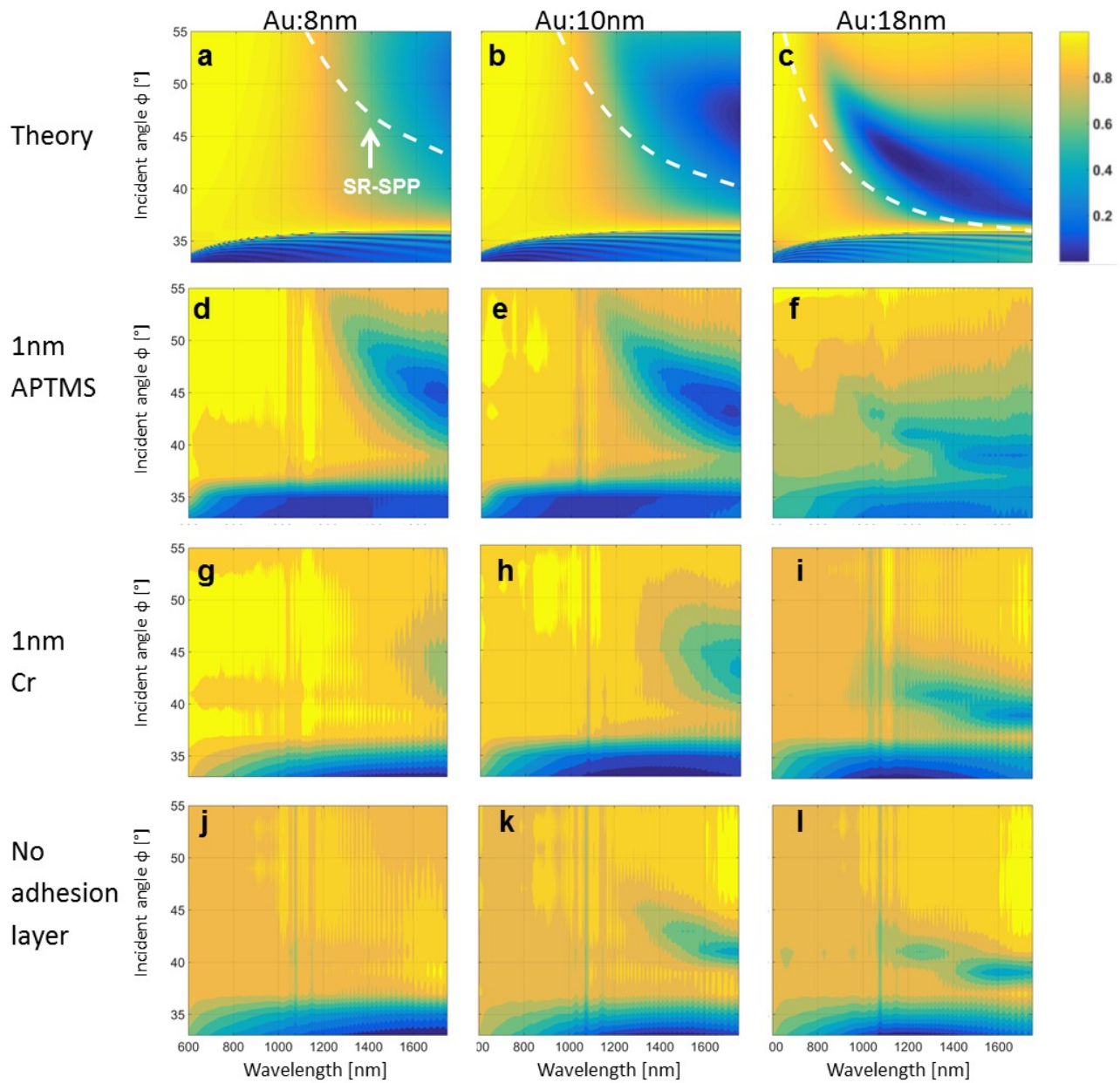

Figure 2. Dispersion diagram of SR-SPPs on ZnSe prism-SiO 2 (200 nm)-adhesion layer (1 nm)-Au-adhesion layer (1 nm)$\mathrm{SiO}_{2}(8 \mu \mathrm{m})-\mathrm{Si}$ substrate structures. Simulations (a-c) and measurements with APTMS adhesion layers (d-f), $1 \mathrm{~nm}$ Cr adhesion layers ( $\mathrm{g}-\mathrm{i}$ ) and without adhesion layer (j-l), for the thickness of $\mathrm{Au}$ 8, 10 and 18 nm, respectively.The white dashed lines in (a)-(c) are theoretical dispersion of SR-SPPs on infinitely thick $\mathrm{SiO}_{2}-\mathrm{Au}-\mathrm{SiO}_{2}$ layers. The critical angle between the $\mathrm{ZnSe}$ prism and the $\mathrm{SiO}_{2}$ layer divides the high and low reflectance regions. 
three different sets of samples: $1 \mathrm{~nm}$ thick APTMS as adhesion layer (figure $2 \mathrm{~d}-\mathrm{f}$ ), $1 \mathrm{~nm}$ thick $\mathrm{Cr}$ adhesion layer (figure 2g-i), and with no adhesion layer (figure 2j-l) compared to theoretical simulations using scattering matrix method considering an ideal uniform Au film embedded in $\mathrm{SiO}_{2}$ (figure 2a-c). The low reflection region beneath 35 degrees is due to the non fulfillment of the total internal reflection conditions below the critical angle between the $\mathrm{ZnSe}$ prism and the $\mathrm{SiO}_{2}$ layer. The oscillations visible in the experimental measurement spectra above 1100 $\mathrm{nm}$ are due to Fabry-Perrot interference in the experimental setup and are not related to the sample's response and the noise close to $1064 \mathrm{~nm}$ visible in the experimental dispersion diagram is due to normalization errors arising from the spectral characteristics of the light source.

For the $8 \mathrm{~nm}$ thick Au film, it can be seen from figure 2d that the experimental dispersion curve for APTMS adhesion layer is much more vivid and reflection dips corresponding to SR-SPPs are clearly visible . However, for the $\mathrm{Cr}$ adhesion layer, the reflectance dip associated with SR-SPPs cannot be observed below $1600 \mathrm{~nm}$ wavelength at all (figure 2g).SR-SPPs suffer the extra damping introduced by the metallic adhesion layer. As a result, SR-SPPs for larger angles of incidence are not supported. For the no adhesion layer, there is no any reflection dip visible for the case of the $8 \mathrm{~nm}$ film (figure $2 \mathrm{j}$ ). This can be understood in terms of the percolation threshold of the $\mathrm{Au}$ film on the $\mathrm{SiO}_{2}$ layer, further sustained by the high Au layer roughness of more than $1 \mathrm{~nm}$ RMS as shown in table 1. In the case of no adhesion layer, the plasmons propagate when the layers start to be continuous around $10 \mathrm{~nm}$ which can be seen from figure $2 \mathrm{k}$.

The role of the adhesion layers become more prominent when the when the thickness of the gold film is below $10 \mathrm{~nm}$ where the SR-SPP is highly localized. However, due to the addition of the lossy Cr metallic adhesion layer, the optical properties of the Au film are deteriorated, which is clearly visible by comparing figure $2 \mathrm{~d}$,e and figure $2 \mathrm{~g}$,h. In comparison with the simulation results, it can be seen that the dispersion curves of ultra-thin Au films with APTMS have better agreement compared to the rest. For example, in the case of $10 \mathrm{~nm} \mathrm{Au}$ film with $\mathrm{Cr}$, the plasmon dips are less pronounced. From this point of view, it is clear that the Au film with APTMS follows more closely the optical characteristics of the ideal Au film. Especially for the thickness of the gold film below $10 \mathrm{~nm}$, the choice of the adhesion layer makes a distinct difference for the existence of SR-SPP modes for the shorter wavelength range and large wavevector region.For the thicker $18 \mathrm{~nm}$ Au layer, the dispersion curves of all the cases looks similar to each other which is due to the fact that the volume ratio of adhesion layer and $\mathrm{Au}$ is low.

\section{CONCLUSION}

We conducted comprehensive experimental and theoretical studies on the influence of adhesion layers on propagating SR-SPPs plasmonic modes on Au films in three configurations: with $\mathrm{Cr}$ adhesion layer, with APTMS adhesion layer and without any adhesion layer. We found that using $\mathrm{Cr}$ as an adhesion layer greatly influences the optical properties of the SR-SPP due to its extra absorptions and scattering. For the Au films with the APTMS adhesion layers, the SR-SPP dispersion follows closely to the one theoretically calculated for completely flat pure Au layers, showing that APTMS is the better adhesion layer between oxide and $\mathrm{Au}$ if one aims to support highly localized propagating plasmon modes.

\section{ACKNOWLEDGMENTS}

This work was supported by Villum Fonden DarkSILD project No. 11116 and Direktr Ib Henriksens Fond, Denmark.

\section{REFERENCES}

[1] Verhagen, E., Polman, A., and Kuipers, L. K., "Nanofocusing in laterally tapered plasmonic waveguides.," Optics express 16(1), 45-57 (2008).

[2] Sreekanth, K. V., Alapan, Y., ElKabbash, M., Ilker, E., Hinczewski, M., Gurkan, U. A., De Luca, A., and Strangi, G., "Extreme sensitivity biosensing platform based on hyperbolic metamaterials," Nature Materials 15, 621-627 (6 2016).

[3] Lu, D., Kan, J. J., Fullerton, E. E., and Liu, Z., "Enhancing spontaneous emission rates of molecules using nanopatterned multilayer hyperbolic metamaterials," Nature Nanotechnology 9(1), 48-53 (2014). 
[4] Leandro, L., Malureanu, R., Rozlosnik, N., and Lavrinenko, A., "Ultrathin, Ultrasmooth Gold Layer on Dielectrics without the Use of Additional Metallic Adhesion Layers," ACS Applied Materials and Interfaces $\mathbf{7}(10)$, 5797-5802 (2015).

[5] Malureanu, R. and Lavrinenko, A., "Ultra-thin films for plasmonics: a technology overview," Nanotechnology Reviews 4, 259-275 (1 2015).

[6] Poddubny, A., Iorsh, I., Belov, P., and Kivshar, Y., "Hyperbolic metamaterials," Nature Photonics 7(12), 948-957 (2013).

[7] Lu, D. and Liu, Z., "Hyperlenses and metalenses for far-field super-resolution imaging," Nature Communications 3, 1205 (2012).

[8] Sukham, J., Takayama, O., Lavrinenko, A. V., and Malureanu, R., "High-quality ultrathin gold layers with an APTMS adhesion for optimal performance of surface plasmon-polariton based devices," ACS Applied Materials 83 Interfaces 9(29), 25049-25056 (2017).

[9] Ko, D. Y. K. and Sambles, J. R., "Scattering matrix method for propagation of radiation in stratified media: attenuated total reflection studies of liquid crystals," Journal of the Optical Society of America A 5(11), 1863 (1988). 